Meta

Journal des traducteurs

Translators' Journal

\title{
Dérivation lexicale et dérive terminologique
}

\section{Pierre Lerat}

Volume 39, numéro 4, décembre 1994

Hommage à Bernard Quemada : termes et textes

URI : https://id.erudit.org/iderudit/003472ar

DOI : https://doi.org/10.7202/003472ar

Aller au sommaire du numéro

Éditeur(s)

Les Presses de l'Université de Montréal

ISSN

0026-0452 (imprimé)

1492-1421 (numérique)

Découvrir la revue

Citer cet article

Lerat, P. (1994). Dérivation lexicale et dérive terminologique. Meta, 39(4),

581-588. https://doi.org/10.7202/003472ar

\section{Résumé de l'article}

La dérivation lexicale n'est pas une opération sémantiquement neutre, du moins dans les vocabulaires techniques, où le " sens linguistique " a souvent peu de rapport avec le " sens terminologique ». La " dérive terminologique » est un effet de la dénomination : elle est un reflet des connaissances non linguistiques, ce qui empêche le mot technique d'être seulement interprétable comme une pure transformation formelle d'un autre mot. L'exemple du verbe passer illustre l'absence de prédictibilité de la forme et du sens des dérivés. On peut imaginer une lexicographie terminologique multidomaine si l'on analyse d'un côté les termes en tant que mots, de l'autre en tant que dénominations de connaissances. Une organisation modulaire des bases de données lexicales le permet. Un essai va être tenté en explicitant les propriétés formelles et les " connectabilités » conceptuelles dans le domaine des contrats. Une utilité des bases de données de cette sorte est de rendre possibles des équivalences conditionnelles pour la traduction : si puce a telle et telle propriété, ou même l'une seulement, alors il faut en anglais chip. On voit aussi que ce travail d'explicitation, suffisant pour la traduction humaine, est le préalable logique à toute automatisation. L'existence de contrôleurs terminologiques est aussi l'une des aides les plus utiles pour la rédaction technique.
Ce document est protégé par la loi sur le droit d'auteur. L’utilisation des services d’Érudit (y compris la reproduction) est assujettie à sa politique d'utilisation que vous pouvez consulter en ligne.

https://apropos.erudit.org/fr/usagers/politique-dutilisation/ 


\title{
DÉRIVATION LEXICALE ET DÉRIVE TERMINOLOGIQUE
}

PIERRE LERAT

Université Paris-Nord, Villetaneuse, France

\begin{abstract}
Résumé
La dérivation lexicale n'est pas une opération sémantiquement neutre, du moins dans les vocabulaires techniques, où le «sens linguistique» a souvent peu de rapport avec le «sens terminologique». La «dérive terminologique» est un effet de la dénomination: elle est un reflet des connaissances non linguistiques, ce qui empêche le mot technique d'être seulement interprétable comme une pure transformation formelle d'un autre mot. L'exemple du verbe passer illustre l'absence de prédictibilité de la forme et du sens des dérivés.

On peut imaginer une lexicographie terminologique multidomaine si l'on analyse d'un côté les termes en tant que mots, de l'autre en tant que dénominations de connaissances. Une organisation modulaire des bases de données lexicales le permet. Un essai va être tenté en explicitant les propriétés formelles et les «connectabilités» conceptuelles dans le domaine des contrats.

Une utilité des bases de données de cette sorte est de rendre possibles des équivalences conditionnelles pour la traduction: si puce a telle et telle propriété, ou même l'une seulement, alors il faut en anglais chip. On voit aussi que ce travail d'explicitation, suffisant pour la traduction humaine, est le préalable logique à toute automatisation. L'existence de contrôleurs terminologiques est aussi l'une des aides les plus utiles pour la rédaction technique.
\end{abstract}

\begin{abstract}
Lexical derivation is not a neutral operation from a semantic point of view, at least in technical vocabulary where "linguistic meaning" has often little to do with "terminological" meaning. The so-called "dérive terminologique" is an effect of the operation of naming: it reflects non-linguistic knowledge, which prevents the technical word from being interpretable only in terms of pure transformation of another word. The example of the verb passer shows a lack of predictibility of both the form and the meaning of the derivatives.

A multifield terminological lexicography can easily be contemplated if terms are analysed on one hand as words, on the other hand as names of knowledge units. A modular organization of lexical databases allows this. This will be tested by explaining the formal properties and the conceptual "connectabilities" in the field of legal contracts.

One use of such data bases is to allow conditional equivalences for translation work: if puce has such or such a property or even only one, then chip is required in English. It is also patently obvious that this explaining work, sufficient for human translation, is a logical precondition for all automation. The existence of terminological checking tools is most useful for technical writing.
\end{abstract}

\section{LE TERME, À LA FOIS MOT ET DÉNOMINATION DE NOTION}

L'opposition entre «définition naturelle» et «définition conventionnelle» chez Robert Martin (1992: 68-69) est très suggestive à un double titre: d'une part elle synthétise le débat sur la nature des mots (phusei vs thesei) en le situant au cour du fonctionnement synchronique et non plus, comme dans la tradition issue de Platon, au niveau très conjectural de l'origine des langues; d'autre part, et c'est ce qui va être retenu ici, elle rend compte de la tension interne qui caractérise tout terme au sens de la terminologie : le terme est à la fois, physiquement, un mot simple ou complexe relevant d'une langue naturelle, et en

Meta, XXXIX, 4, 1994 
même temps, sémiotiquement, la dénomination conventionnelle d'une notion technique dans un milieu professionnel.

La dérivation lexicale, au même titre que la composition (notamment syntagmatique), est à l'origine de beaucoup de termes, y compris néologiques (voir Quemada 1983). Ceux-ci sont quelquefois physiquement marqués, comme les noms des produits chimiques, mais le plus souvent ils utilisent les ressources ordinaires de la morphologie lexicale; parmi ces derniers, on s'en tiendra ici à des cas de morphologie non savante (en laissant de côté les formants latins et grecs étudiés par $\mathrm{H}$. Cottez), propres à inciter à la prise en compte d'un «sens linguistique, qui correspond à une paraphrase minimale du mot construit à l'aide d'un mot de la même famille» (Lerat 1984: 160-161). Ce sens est le signifié saussurien, valeur de radical historique mémorisé, à la base de la continuité de la langue, de la bonne formation lexicale et de l'organisation du dictionnaire de langue.

Le terme résultant d'une dérivation lexicale endogène (pour ce qui est du cas des emprunts, voir Lerat 1988) est également susceptible, comme les autres, d'une prise en considération au titre d'un «sens terminologique» (Lerat 1984: 161), c'est-à-dire par rapport à des connaissances extralinguistiques. La lexicographie est prise entre les deux exigences : tenir compte de la motivation morphologique du dérivé, mais aussi du sens technique (voir Guilbert 1981) qui constitue la «valeur d'emploi» (Rey 1985:6) normale du mot spécialisé, sa dénotation analysable en termes de conditions nécessaires et suffisantes, telle que la reflète le jeu des relations de dépendance syntaxique dans les énoncés spécialisés; ainsi, le sens utile d'action en matière boursière n'est pas l'idée d'agir mais un ensemble de propositions analytiques telles que «est un titre négociable», «est émis par une société» et «constate le droit de l'associé dans la société» (voir Lerat 1993).

\section{LA DÉRIVE TERMINOLOGIQUE DE «LUNETTE» ET DE «LENTILLE»}

Les terminologies sont dans les langues (voir Quemada 1978), mais aussi dans les cultures. Au titre du français, lunette est le nom d'un «objet en forme de lune» depuis 1200 environ, lentille celui d'un "verre en forme de lentille», comme le dit excellemment Descartes (voir le Trésor de la langue française, ci-après appelé T.L.F.); ces analogies sont dans la logique des séries associatives saussuriennes. Techniquement, en revanche, et sans donner à technique une connotation d'ésotérisme, d'autant qu'il s'agit de l'expérience quotidienne d'un objet familier, tout éloigne lunette de lune, en dehors de la dérivation suffixale: le paradigme dérivationnel pertinent (à sens constant de la base), la syntagmatique et le sens conceptuel (la définition).

Morphologiquement, lunette a un rapport historique avec lune, mais pour un opticien et pour son client, dans leur interaction normale, la dérivation lexicale pertinente sera celle qui prend comme base lunettes et qui justifie les définitions dérivationnelles du T.L.F. selon lesquelles la lunetterie est l' ' industrie des lunettes» et le lunetier «celui, celle qui fabrique ou qui vend des lunettes». Syntaxiquement, lunettes sera anaphorisable par instrument, non par satellite; il pourra dépendre d'un verbe approprié (comme sujet ou comme complément de moyen, si c'est corriger, comme complément d'objet direct si c'est porter), ou d'un substantif ad hoc (verre ou monture). J'appelle dérive terminologique ce phénomène banal par lequel la logique des connaissances s'impose dans le discours au détriment de la logique de la langue.

La dérive terminologique est sanctionnée dans la définition, y compris dans le dictionnaire de langue, qui présente les lunettes comme un «instrument d'optique composé d'une paire de verres ou de disques d'une autre matière transparente, enchâssés dans une monture que l'on place sur le nez et devant les yeux pour corriger la vue ou protéger les yeux» (T.L.F.). De même, la lentille n'a plus rien de la «plante légumineuse» nommée 
ainsi depuis le XIIème siècle au moins, par évolution phonétique régulière à partir du latin populaire lenticula: en revanche, elle a des propriétés distinctives qui l'apparentent aux lunettes et l'en distinguent à la fois, puisque c'est un «petit disque bombé en matière plastique, qui s'applique sur la cornée pour corriger certains défauts des yeux» (T.L.F.). Dans un dictionnaire d'optique, on n'aurait pas à rendre compte de la dérive terminologique de lentille, ni non plus de lunette, mais il est bon que le dictionnaire général unilingue établisse un pont («par analogie de forme») entre termes, en rappelant qu'il s'agit bien d'un même signe linguistique saussurien.

La dérive terminologique est perturbatrice pour la communication en langue étrangère: lunettes de soleil se dit en anglais sunglasses, mais le nom de la lunette d'un fusil est sight, la lunette arrière d'une voiture s'appelle rear window, un lunetier est un spectacle manufacturer, bref les aléas de la dérivation et de la composition dans chaque langue contribuent au désordre lexical dans la communication internationale. Double aliénation : loi d'un autre arbitraire du signe, loi de l'étiquetage au gré de «l'expérience de la praxis humaine» (Quemada 1978: 1149). Triple aliénation, si l'expérience elle-même fait défaut, comme c'est le cas pour le non-spécialiste de connaissances pointues, qui sera incapable de conceptualiser, donc d'intégrer à sa culture, ce qu'il percevra comme simple nomenclature, comme jargon ou comme snobisme, en tout cas comme une bizarrerie.

\section{FAMILLE DE MOTS ET CHAMP NOTIONNEL}

Par rapport à un mot polysémique comme passer, la liste de ses dérivés donne jusqu'à un certain point l'impression d'un ordre de la langue; plus précisément, celle d'une motivation relative, au sens saussurien. Continuité des signifiants : passage, passation, passe, passée et passement, formés comme d'autres noms d'actions du français, passant et passeur pour les noms d'agents. Continuité des signifiés : chacun des dérivés correspond plus ou moins à un sens minimal tel que «x va de $A$ à $B »$, en français (dans une autre langue il faudrait recourir à plusieurs radicaux, mais le signifié est immanent, donc ne sortons pas du cadre de la famille morphologique du verbe français passer).

La dérive terminologique ressort bien, en revanche, de l'analyse transformationnelle: comment prédire quelle nominalisation convient aux bécasses, aux actes juridiques, aux tissus? Dire que passée suppose une phrase de base avec un sujet qui soit animé et passation un objet inanimé ne fait pas beaucoup progresser la traduction ou la génération automatique. En revanche, affiner les critères de sélection fait voir la réalité des conventions terminologiques et, plus largement, dénominatives; de même, l'inventaire des paraphrases permettant de rendre compte d'un nom d'agent dérivé dit quelque chose sur son sens : un commerçant n'est pas seulement quelqu'un qui fait du commerce, mais qui est dans le commerce ou qui tient un commerce, la dénomination épousant la polysémie de commerce (voir Lerat 1984: 28).

Si l'on gagne peu à appliquer aux dérivés des traits de sous-catégorisation tels que animé, comptable et autres du même genre, la détermination de classes pragmatiques permet des prédictions utiles pour la rédaction et la traduction assistées. L'idée de «connectabilités» conceptuelles (Picht) est conforme à la réalité de la dérive terminologique: passée des bécasses et passation du contrat condensent syntaxiquement des propositions plausibles, contrairement à passée des vaches, passée du contrat, etc.

Cette terminologisation croissante des langues (aussi vieille que la civilisation, comme on le voit dans les travaux de Benveniste sur le vocabulaire en indo-européen, mais particulièrement sensible dès les débuts de l'industrialisation, comme le montre la thèse de R. Eluerd), ne conduit pas pour autant à une marginalité du terminologique : les termes font tout à fait partie de la langue, y compris s'ils sont empruntés. Ils s'écrivent, se 
prononcent, ont une flexion, des dérivés, des composés, ils obéissent à la syntaxe. En revanche, la dérive terminologique se mesure à l'imprévisibilité plus ou moins grande des définitions des mots technicisés. Plus précisément, ces définitions prennent appui sur des champs notionnels: lunettes renvoie à corriger (dans un sens technique), à verres (au sens des instruments d'optique), à monture (de lunettes), etc. Ces champs notionnels ne sont pas des champs lexicaux (reflétant le «sens naturel») mais des champs de dénominations (substantivales, verbales; adjectivales et adverbiales) de concepts. Il ne s'agit nullement de la lune, mais de liens tels que «sorte de» instrument, «partie de» (applicable à monture et à verre) et de relations fonctionnelles à pertinence syntaxique (expressions dépendantes comme de soleil et arrière, expressions régissantes comme corriger). La limitation des compatibilités acceptables constitue en effet un indice privilégié de la terminologisation (voir Lerat 1993).

\section{LA DÉRIVE TERMINOLOGIQUE À PARTIR DU VERBE «PASSER»}

Le français a plusieurs suffixes de noms d'agents, dont l'un, -eur, appliqué à la base verbale passe, est susceptible de donner un nom à la fois à celui qui passe et à celui qui fait passer, que ce soit occasionnellement ou professionnellement. C'est aussi le nom du «joueur qui fait des passes» (T.L.F.), au sens répertorié sous le domaine SPORTS. Mais il existe aussi des centaines de noms d'agents en -ant, dont un curieux passant qui a l'infirmité de ne se déplacer qu'à pied (sinon c'est un passager, du moins s'il ne conduit pas lui-même).

Du côté des noms d'actions, l'anomie linguistique et l'arbitraire terminologique sont plus spectaculaires encore. La passation régit une série très restreinte d'arguments pragmatiquement acceptables, en tant qu' «action de dresser, d'établir (un acte, un contrat» (T.L.F.). Le passement est un «tissu de fils mêlés servant d'ornement» (ibid.), la passée le «passage habituel du gibier d'eāu, des bécasses (...)» (ibid.), selon une monosémisation qui résulte de la disparition des emplois moins spécifiques. Avec passage, c'est la distribution qui rend la communication claire: avis de passage va avec la mention de domaine POSTES, droit de passage avec DROIT, passage des vitesses avec AUTOM., examen de passage avec ENSEIGN., passage à l' acte avec PSYCHOL.; au sein même de la rubrique SPORTS, elle conduit à séparer le temps de passage (du coureur) et le passage du témoin (par le coureur) : quelle belle machinerie qu'une langue naturelle!

Avec passe, dérivé régressif qui sent son ancienneté et qui n'a plus que des emplois professionnels (y compris le «rapport sexuel rapide d'une prostituée avec son client»), la dérive terminologique conduit à une démotivation morphologique par rapport à passer. La liste d'emplois ci-dessous, tirée du T.L.F., se passe de commentaire (à ceci près que c'est moi qui souligne les arguments et prédicats requis):

MÉCAN. : «Action par laquelle on soumet une pièce ou un matériau au travail exécuté par un outil sur une machine-outil en un cycle mécanique»

GYMN.: «Passage d'un acrobate (...)»

SPORTS : «Action du joueur (...)»

TAUROM. : «Passe de cape (...)»

OCCULT. : «Geste des mains (...)»

CHAPELL. : «Bord d'un chapeau de femme (...)»

Le plus remarquable est que cette diversification pragmatique est un phénomène incessant, comme le montre le Dictionnaire de termes nouveaux des sciences et des techniques (Quemada 1983:221):

EXPLOITATION DES RESSOURCES MINÉRALES : «Ouverture étroite et longue qui permet l'exploitation d'une mine»

PÊCHE: Passe à poissons: «Canal ou orifice ménagé dans un barrage pour permettre le passage habituel des poissons» 


\section{COMMENT TRAITER TERMINOLOGIQUEMENT CE QUI EST TERMINOLOGIQUE ?}

Une lexicographie terminologique multidomaine est possible à une condition: si elle fait une place aux termes à la fois en tant que mots et en tant que dénominations de connaissances. Le support électronique rend possible une navigation entre fiches de mots et fiches de dénominations (nominales, verbales, adjectivales et adverbiales) pour peu qu'elles soient susceptibles d'une unification par le lemme, entrée obligée des unes et des autres (voir Lerat 1994). Le tout en un ne s'impose plus dès lors qu'une organisation modulaire permet d'éviter trois écueils: celui du dictionnaire de langue générale, qui ne connaît de connaissances non linguistiques qu'en miettes, celui du dictionnaire spécialisé, qui réserve le même sort aux connaissances linguistiques, celui de la banque de données terminologique multidomaine classique, qui juxtapose des fiches de traducteurs sans systématisation linguistique ni cognitive. La formule la plus cohérente, la plus prévisible et donc la plus utile devrait être l'explicitation, séparément, des relations linguistiques formelles (morphologiques et syntaxiques) et des relations conceptuelles (matérialisées par les dépendances syntaxiques propres à telle acception); ces relations conceptuelles, au reste, ne doivent pas être idéalisées: elles n'existent que dans la mesure où elles sont gagées sur des «transactions linguistiques réelles» (L. Wittgenstein cité in $\mathrm{R}$. Eluerd 1993: 386).

A titre d'exemple, les valeurs techniques de passage (en matière de courses, de psychologie, de transports, de mécanique, de droit, etc.) seraient susceptibles de traductions non aléatoires au prix de deux fichiers mis en relation, pour chaque langue traitée. Dans le fichier de mots, l'entrée serait accompagnée d'informations consistant en les caractéristiques morphologiques du mot, en la ou les distributions usuelles, en une ou des définitions minimales, et en renvois à chacune des fiches terminologiques correspondantes. Dans le fichier de termes, le même lemme aurait autant d'entrées que d'emplois terminologisés, et chaque fiche pourrait comporter le paradigme dérivationnel ad hoc (voir Chabridon et Lerat 1993), les compléments et régissants syntaxiques requis pragmatiquement, un exemple indicatif, le cas échéant un antonyme, autant que possible un hyperonyme strict, nécessairement le domaine prototypique (à titre de repère, même grossier), une définition, une note, des sources autorisées, les équivalents conditionnels dans les autres langues de la base (voir Lerat 1994).

La fiabilité d'une double base de cette sorte et ses possibilités réelles d'exploitation (industrielle ou artisanale) n'ont jamais été explorées, à ma connaissance. Pour commencer, et à titre de prototype, la matière d'un Dictionnaire juridique (Sourioux et Lerat 1994) va être soumise à une expérience de traitement modulaire sur base de données relationnelle au prix d'un effort accru d'explicitation des propriétés formelles (y compris dans la partie terminologique, puisque chaque acception a ses dérivés et ses composés propres) et des compatibilités pragmatiques (classes de régissants et dépendants requis), le but étant de fournir une assistance au traducteur spécialisé (pour qui le sans-faute passe par l'équivalence conditionnelle) et au rédacteur (qui n'a pas seulement à se soucier de collocations, mais d'abord de graphie, de flexion, de prépositions, d'articles et d'auxiliaires).

\section{ENTRE LE CALCULABLE ET LIMPRÉVISIBLE, LE REPÉRABLE}

Les lexicographes ont beau faire, l'article passer dans tout dictionnaire est plus ou moins chaotique. Ce qui tempère le désordre, dans le meilleur cas (voir Gorcy 1990) est la soumission à un cahier des normes; toute tentative pour affiner les critères risque d'être $a d$ $h o c$, que l'on donne priorité à une ordonnance fondée sur la distribution (voir Dubois 1962) ou que l'on cède à la séduction d'une sémantique «profonde» (voir Lerat 1987).

La part du terminologique, c'est-à-dire de l'ordre des connaissances, dans les causes de cet éclatement des informations portant sur un mot et sur ses dérivés, rend aussi illusoires les prédictions syntactico-sémantiques reposant sur les traits popularisés par les 
grammaires génératives et post-génératives (tout passe: l'humain comme le non-humain, l'inanimé comme l'animé, l'abstrait comme le concret, le compact et le non compact) et l'idée même d'un «sens dérivationnellement prédictible» (Corbin 1991: 11). Dès lors que l'on entre dans l'art des «adaptations pragmatiques du sens prédictible» (ibid.: 12), la référence à une «structure morphologique profonde» (p. 17) n'est pas plus explicative que sa concurrente l'étymologie, tout en étant moins falsifiable. Ce qui rend néanmoins séduisant le recours à un «modèle associatif et stratifié» est le cas où la dérive terminologique peut être minimisée par la technique lexicographique de la définition dérivationnelle (balayer, balayage, balayeur employé de la voirie, balayeuse munie d'une brosse rotative: les spécifications s'accumulent au cours de la dérivation, mais l'opération concernée tient toujours du coup de balai), dont le rendement est souvent appréciable (voir Martin 1992: 63) tant que l'information attendue se limite à une définition «naturelle». Ce qui fait tort à son crédit, inversement, c'est un cas comme celui de passe, où le scénario du footballeur est «spécial» et appelle une définition «conventionnelle».

Le traducteur, qui compte sur le dictionnaire unilingue pour valider les informations puisées dans le bilingue, n'a ni le temps de faire un parcours qui n'est pas le sien (celui de la logique lexicographique) ni même intérêt à bénéficier d'un cheminement «du plus régulier au moins régulier» (Corbin 1991: 18). En revanche, il attend des repères: un domaine, une classe d'objets appropriés (voir Gross 1992), la préposition et l'article requis, etc. C'est un tel jeu de coordonnées qui rend possible des équivalences conditionnelles.

\section{VERS L'ÉQUIVALENCE CONDITIONNELLE}

La recherche de l'équivalent est une quête mythique en langue générale, mais elle a de bonnes chances de succès là où les mots sont les vecteurs de connaissances partagées (pharmacie, machine-outil, droit international, informatique, etc.). Si une puce est une microplaquette électronique, si elle est désignée dans un texte relevant de l'électronique, si l'on parle à son propos de composants ou de circuit intégré, alors le mot anglais équivalent est chip; en fait, en l'occurrence un seul de ces trois critères suffit, mais la sécurité passe par la multiplicité des modes de contrôle; aussi bien, la mention SPORTS ne sera pas suffisamment discriminante dans un cas comme celui de passe (qui a un usage sensiblement différent au football et en matière d'escrime). Comment automatiser 1'accès à l'équivalent correct en langue étrangère?

Pour le traducteur humain, une ressource qui a fait ses preuves dans la gestion de données de toutes sortes est la possibilité de tris multicritères. Tant mieux si le domaine suffit, mais c'est rarement le cas, parce que les noms de domaines sont eux-mêmes des termes, donc des mots sujets à la polysémie, la polyhiérarchie et la quasi-synonymie; mais si j'ai à traduire passage au sens philologique il reste que littérature fera l'affaire (pour une machine, une approximation comme écrit ne sera pas exploitable). Le paradigme dérivationnel est un repère qui peut également avoir son prix; ainsi, la série passer-passagepasseur restreint les équivalents possibles. Les arguments appropriés sont plus ou moins discriminants : la passée est avant tout le fait des bécasses, dans ma région, mais ailleurs on observe des emplois moins exclusifs, à propos d'autres oiseaux ou d'animaux en général (voir le T.L.F.). Un exemple peut éclairer, à la façon des «syntagmes» du T.L.F.: passeur de cocaine est prototypique d'une acception de passeur. Les hyperonymes guident également l'interprétation préalable à la traduction, en dépit des polyhiérarchies (un passage est tantôt une action, tantôt un lieu). Une source autorisée est à tout le moins une caution sécurisante, une définition a l'intérêt d'expliciter l'autonomie relative de l'acception. Si rien de tout cela n'est nécessaire ni suffisant, un faisceau de plusieurs indicateurs tels que ceux-ci, qui appartiennent à la meilleure tradition lexicographique, rend plausible une équivalence conditionnelle du type suivant: 
si $\{$ lemme $\mathrm{fr}\}=$ passe \& $\{$ domaine $\}=$ sports \& $\{$ dépendant $\}=$ joueur, ballon \& [régissant $=$ envoyer, alors $\{$ lemme en $\}=$ pass $\&\{$ lemme de $\}=$ Abgabe, etc.

Dans le cas de l'assistance à la traduction humaine, qui est une étape dont on ne saurait faire l'économie puisque c'est celle de l'explicitation des faits de langue au fur et à mesure du traitement des difficultés réelles, qui est logiquement requise en amont de leur modélisation et non pas en aval, l'homonymie de passe est un problème sans acuité. En revanche, il faut une gestion économique des particularités linguistiques et une explicitation des connaissances non linguistiques à un niveau réellement professionnel. Le premier aspect suppose que le travail soit assuré ou révisé en partie par des linguistes de métier, qui signalent les constructions usuelles des mots en tant que tels, une seule fois pour chaque forme polysémique. Le second exige la participation de spécialistes humanistes, qui aient le talent de rendre compréhensible ce qu'ils savent.

La néologie technique est à cet égard un excellent observatoire des problèmes posés par la dérive terminologique. Un autre révélateur est le sort des néologismes d'hier et d'avant-hier, qui incite à la circonspection. Un témoignage à méditer est celui de la belle thèse de R. Eluerd, où l'on voit à quel point la sidérurgie a été consommatrice de mots, bien ou mal formés. Le terme acier, qui a été le nom d'un concept longtemps hésitant avant que les spécialistes se mettent d'accord sur les propriétés nécessaires et suffisantes de cet alliage de fer et de carbone, a donné lieu à une dérivation qui rétrospectivement peut paraître luxuriante mais qui est née de besoins de nomination et de distinction multiples, en relation avec les dialectes et les milieux de travail.

La sidérurgie a vu naître et mourir acérage, acérain, acération, acérin, acérure, acerie, aciération, aciéreux, aciéron, ascierie et aciron. Acérain est dans le T.L.F., mais uniquement grâce à la prise en compte du supplément du dictionnaire de l'Académie de 1798, à côté d'acéreux («allongé, pointu et dur», du latin acerosus!), d'acéride («emplâtre dans la composition duquel il n'entre pas de cire»!), d'acériné («qui ressemble à l'érable»!) et d'acérique (extrait de ... l'érable!); acérure aussi, mais sans attestation au-delà de Littré, de même qu'aciéreux; aciération n'est mentionné que par égard pour le travail des académiciens, et les autres formes ont disparu des dictionnaires eux-mêmes.

À quoi se réduit le paradigme dérivationnel vivant d'acier en 1994? Il nous reste aciérage, qui a supplanté acération et acirerie (Eluerd 1993: 90), aciériste (qui l'a emporté sur aciéreur), aciérie (qui a longtemps désigné l'opération d'aciérage, en même temps que le «fourneau d'aciérage») et acérer. Encore la dérive terminologique a-t-elle affecté ce verbe: on part d'un sens professionnel («mettre de l'acier avec du fer»), puis il acquiert un sens technique bien différent («convertir en acier»). Après quoi l'imaginaire collectif a été le plus fort: c'est effectivement «par analogie» ou «au figuré» que l'on juge normalement («en langue») acérés une flamme, un feuillage, une rumeur, une note, une idée, une science, une parole, un sourire, un philosophe (voir le T.L.F.).

\section{POUR DES CONTRÔLEURS TERMINOLOGIQUES}

Entre les dictionnaires de langue traditionnels (y compris sur support électronique) et les dictionnaires électroniques au sens de $M$. Gross, il y a place, sans doute pour longtemps, pour des instruments d'aide à la rédaction (en une ou plusieurs langues) visant à rendre prévisibles la maîtrise de «transactions linguistiques réelles». C'est en principe ce qu'on attend des banques de données terminologiques, mais celles qui sont consultables commodément, c'est-à-dire grâce à un lecteur de CD-ROM, sont loin de répondre à des attentes légitimes comme les suivantes:

- une explicitation suffisante des contraintes linguistiques;

- une explicitation suffisante des connaissances non linguistiques qui constituent le mode d'emploi des termes; 
- la synthèse en une seule fiche «à jour pour chaque notion» au lieu d'une «masse d'attestations» (Lerat et Budin 1992 : 147).

Les «transactions linguistiques réelles» reposant sur des conventions pragmatiques, on ne peut attendre l'impossible de la morphologie, ni de la syntaxe, ni de la sémantique componentielle. C'est ce que montre bien la contribution de B. Quemada à 1'Histoire des techniques, par exemple à propos des dérivés d'usine: «seules des conventions nées de l'usage permettent d'éviter la confusion entre usinier = industriel et usineur = ouvrier spécialisé» (1978: 1192). Cette absence de prédictibilité est en outre confirmée par l'apparition d'un autre emploi d'usineur, «machine-outil servant à façonner des pièces» (Quemada 1983: 221). Ainsi, les attentes courantes des rédacteurs et des traducteurs techniques dans les deux sens (onomasiologie et sémasiologie) incitent à souhaiter l'élaboration de contrôleurs terminologiques à double entrée, une par le mot et une par la notion, en utilisant comme passerelle la double nature du terme: signe linguistique et dénomination de connaissances. Sinon, comment maîtriser, dans chaque langue, à la fois les aléas de la polysémie et les contraintes de la monoréférence?

\section{RÉFÉRENCES}

BENVENISTE, E. (1948) : Noms d'agent et noms d'action en indo-européen, Paris, Adrien-Maisonneuve.

CHABRIDON, J. et P. LERAT (1993): «Terme et famille de termes», La banque des mots, $\mathrm{n}^{\circ} 5$, pp. 55-63.

CORBIN, D. (1991) : «Introduction. La formation des mots : structures et interprétations», Lexique, $\mathrm{n}^{\circ}$ 10, pp. 7-30.

COTTEZ, H. (1980) : Dictionnaire des structures du vocabulaire savant, Paris, Le Robert.

DUBOIS, J. (1962): «Recherches lexicographiques : esquisse d'un dictionnaire structural», Études de linguistique appliquée, $\mathrm{n}^{\circ} 1, \mathrm{pp} .43-48$.

ELUERD, R. (1993) : Les mots du fer et des lumières, Paris, H. Champion.

GORCY, G. (1990) : «La polysémie verbale ou le traitement de la polysémie de sens dans le Trésor de la langue française : discussion à partir des normes rédactionnelles», Cahiers de lexicologie, $\mathrm{n}^{\circ}$ 56-57, pp. 109-122.

GROSS, G. (1992) : «Forme d'un dictionnaire électronique», L'environnement traductionnel : la station de travail du traducteur de l'an 2001, Actes des Deuxièmes Joumées scientifiques du réseau LTT de l'AUPELFUREF, Sillery, Presses de I'Université du Québec, pp. 255-271.

GUILBERT, L. (1981): «Les relations entre l'aspect terminologique et l'aspect linguistique du mot», Textes choisis de terminologie. G. Rondeau et H. Felber (Eds.), Québec, GIRSTERM, Université Laval, pp. 187-197.

KALONJI, M. T. (1993) : La lexicographie bilingue en Afrique francophone. L'exemple français-cilubà, Paris, L'Harmattan.

LERAT, P. (1984) : «Grammaire des noms d'agents en -ANT en français contemporain», Cahiers de lexicologie, $\mathrm{n}^{\circ} 1$, pp. 23-39.

LERAT, P. (1987) : «L'acceptabilité des mots», Études de linguistique appliquée, $\mathrm{n}^{\circ}$ 67, pp. 7-34

LERAT, P. (1988) : «Les internationalismes dans les langues romanes», Hommage à Bernard Pottier, vol. 2 , pp. 483-491.

LERAT, P. (1993) : «La représentation des connaissances en terminologie assistée», à paraître dans les actes du colloque Traductique de Montréal.

LERAT, P. (1994) : Les langues spécialisées, Paris, Presses Universitaires de France (sous presse).

LERAT, P. et G. BUDIN (1992) : «La formation à la rédaction technique assistée», TAMA'92, Vienne, TermNet, pp. $145-155$.

LERAT, P. et J. L. SOURIOUX (1994) : Dictionnaire juridique. Terminologie du contrat (en français, avec des équivalents en anglais et en allemand), Paris, Conseil international de la langue française.

MARTIN, R. (1992) : Pour une logique du sens (1983), $2^{\mathrm{e}}$ éd., Paris, Presses Universitaires de France.

PICHT, H. (1990) : «LSP Phraseology from the Terminological Point of View», Terminology Science and Research (Vienne, TermNet), vol. I, $\mathrm{n}^{\circ} 1-2$, pp. 33-48.

QUEMADA, B. (1978) : «Technique et langage», Histoire des techniques, B. Gille (Éd.), Paris, Gallimard, pp. $1146-1240$.

QUEMADA, G. (1983) : Dictionnaire des termes nouveaux des sciences et des techniques, Paris, Conseil international de la langue française et Agence de coopération culturelle et technique.

REY, A. (1985) : «La terminologie dans un dictionnaire général de la langue française : le Grand Robert», TermNet News, $\mathrm{n}^{\circ}$ 14, pp. 5-7. 